IZA DP No. 5599

Ökonometrie vs. Projektdesign:

Lehren aus der Evaluation eines Modellprojekts zur Umsetzung des Workfare-Konzepts

Hilmar Schneider

Arne Uhlendorff

Klaus F. Zimmermann

March 2011 


\section{Ökonometrie vs. Projektdesign: Lehren aus der Evaluation eines Modellprojekts zur Umsetzung des Workfare-Konzepts}

Hilmar Schneider

IZA und DIW Berlin

Arne Uhlendorff

Universität Mannheim, IZA und DIW Berlin

Klaus F. Zimmermann

IZA und Universität Bonn

Discussion Paper No. 5599

March 2011

IZA

P.O. Box 7240

53072 Bonn

Germany

Phone: +49-228-3894-0

Fax: +49-228-3894-180

E-mail: iza@iza.org

Any opinions expressed here are those of the author(s) and not those of IZA. Research published in this series may include views on policy, but the institute itself takes no institutional policy positions.

The Institute for the Study of Labor (IZA) in Bonn is a local and virtual international research center and a place of communication between science, politics and business. IZA is an independent nonprofit organization supported by Deutsche Post Foundation. The center is associated with the University of Bonn and offers a stimulating research environment through its international network, workshops and conferences, data service, project support, research visits and doctoral program. IZA engages in (i) original and internationally competitive research in all fields of labor economics, (ii) development of policy concepts, and (iii) dissemination of research results and concepts to the interested public.

IZA Discussion Papers often represent preliminary work and are circulated to encourage discussion. Citation of such a paper should account for its provisional character. A revised version may be available directly from the author. 
IZA Discussion Paper No. 5599

March 2011

\section{ABSTRACT \\ Ökonometrie vs. Projektdesign: Lehren aus der Evaluation eines Modellprojekts zur Umsetzung des Workfare-Konzepts ${ }^{*}$}

We evaluate a pilot workfare project of a Berlin city community to integrate young people in social assistance into the labor market. Reference data are generated in collaboration with the German Employment Office. The participation effect is found to be positive and of relevant size, but not statistically significant. This indicates that selection biases detected in the allocation process of individuals to the program can be adjusted by econometric techniques, but only on the prize of reduced significance levels. Given the small sample sizes of pilot projects this calls for social experiments in controlled project designs.

JEL Classification: J22, J64, H43

Keywords: $\quad$ workfare, labor supply, evaluation, social experiments

Corresponding author:

Hilmar Schneider

IZA

Schaumburg-Lippe-Str. 5-9

53113 Bonn

Germany

E-mail: schneider@iza.org

\footnotetext{
* Die Autoren danken der Deutschen Forschungsgemeinschaft (DFG) für finanzielle Unterstützung im Rahmen des Schwerpunktprogramms „Flexibilisierungspotenziale bei heterogenen Arbeitsmärkten“, Teilprojekt ZI 302/7-1. Darüber hinaus danken wir den Mitarbeiter des Sozialamtes BerlinCharlottenburg sowie des Instituts für Arbeitsmarktforschung und Berufsforschung für ihre Unterstützung und Jan Stuhler für seine Mitarbeit. Wir danken ferner Friedrich-Wilhelm Dopatka, ehemals Staatsekretär im Berliner Senat, für seine Hinweise auf das dieser Studie zugrunde liegende Modellprojekt.
} 


\section{Mangelnder Erkenntnisfortschritt in der Praxis von Modellprojekten}

Die hohe Zahl von arbeitslosen Sozialhilfebeziehern bzw. Beziehern von Leistungen der Grundsicherung ist für die Politik bereits seit geraumer Zeit Anlass, nach Konzepten Ausschau zu halten, mit denen sich einerseits die hohen Belastungen für die öffentlichen Haushalte reduzieren lassen und andererseits den Betroffenen eine Perspektive für ein unabhängiges Einkommen vermittelt werden kann. In zahlreichen Modellversuchen auf lokaler und überregionaler Ebene ist bislang versucht worden, das Problem zu überwinden. Umso mehr erstaunt es, dass sich aus dieser Praxis bislang kein durchschlagendes Lösungskonzept herauskristallisiert hat. Die Ursache dafür dürfte vor allem darin zu suchen sein, dass die bisherigen Modellversuche kaum dokumentiert sind und von ihrer Umsetzung her nur in Ausnahmefällen darauf angelegt waren, belastbare Schlussfolgerungen zu ziehen. Häufig mangelt es aus Kostengründen an Informationen über Erwerbsverläufe im Anschluss an die Programmteilnahme, ohne die keine valide Beurteilung der mittel- und langfristigen Effekte eines Programms möglich ist. Hinzu kommt, dass Modellversuche häufig nur mit eher geringen Fallzahlen durchgeführt werden, was das Auffinden statistisch signifikanter Effekte erschwert. Das gilt vor allem dann, wenn die Teilnahme an einem Projekt selektiv erfolgt. Es besteht demnach eine beträchtliche Diskrepanz zwischen dem enormen Aufwand an öffentlichen Mitteln, der mit der Durchführung von Modellversuchen verbunden ist, und dem Wissen über die Wirksamkeit der durchgeführten Maßnahmen.

Der vorliegende Beitrag zeigt am Beispiel eines konkreten Modellprojekts auf, wie die geschilderten Probleme prinzipiell überwunden werden können. Es handelt sich um das Projekt „Arbeit Sofort!“, das vom Herbst 2002 bis zum Sommer 2003 im Berliner Bezirk Charlottenburg-Willmersdorf durchgeführt wurde. Die Zielgruppe bestand aus Sozialhilfeempfängern im Alter von 18 bis 25 Jahren. Insbesondere für Neuantragsteller auf Sozialhilfe wurde der Leistungsbezug an die Aufnahme einer Tätigkeit im Rahmen eines öffentlich geförderten Beschäftigungsprojekts gebunden. Das Projekt folgte somit dem WorkfareKonzept mit dem Ziel, die Erwerbsanreize für Niedrigqualifizierte durch eine Koppelung von Leistungsansprüchen an eine Gegenleistung in Form von Arbeit zu erhöhen (vgl. Bonin et al. 2007).

Das Ziel der Evaluation dieses Projekts besteht in der Ermittlung des kausalen Effekts der Teilnahme an „Arbeit sofort!“ auf die Beschäftigungswahrscheinlichkeit der Teilnehmer. 
Dazu werden die Erwerbsverläufe der Gruppe der Programmteilnehmer mit den Erwerbsverläufen einer geeigneten Gruppe von Sozialhilfebeziehern verglichen, die nicht an dem Programm teilgenommen haben. Die erforderlichen Daten wurden durch eine anonymisierte Verknüpfung der Personendaten aus dem Sozialamt mit Geschäftsdaten der Bundesagentur für Arbeit gewonnen. Diese Vorgehen generiert nicht nur äußerst verlässliche Daten, sondern vermeidet zugleich auch die nicht unerheblichen Befragungsausfälle, die ansonsten bei der spezifischen Zielgruppe zu erwarten gewesen wären.

Ein typisches Problem für die Programmevaluation resultiert aus einem selektiven Maßnahmenzugang, sowohl durch Selbstselektion der Teilnehmer als auch durch selektive Maßnahmenzuweisung seitens der Mitarbeiter der Sozialbehörde. Selektionseffekte verhindern, dass sich Ergebnisunterschiede zwischen Programmteilnehmern und Vergleichsgruppe ausschließlich auf die Programmteilnahme zurückführen lassen. Da sich eine rein zufällige und damit nicht selektive Programmteilnahme im vorliegenden Fall nicht realisieren ließ und die Selektion im Zuweisungsverfahren für uns nicht kontrollierbar war, sind wir darauf angewiesen, im Nachhinein entsprechende Selektionskorrekturen mit Hilfe statistischer Modelle vorzunehmen. Allerdings basieren alle einschlägigen Ansätze letztlich auf bestimmten Annahmen, die nicht überprüft werden können. Für einen methodischen Überblick siehe bspw. Lee (2005), Blundell und Costa Dias (2009) oder Imbens und Wooldridge (2008). Der Preis für die Verwendung von Verfahren, bei denen zusätzlich der Selektionsprozess in die Maßnahme modelliert wird, besteht neben der Abhängigkeit von der Gültigkeit der getroffenen Annahmen in einer erhöhten Parameterstreuung, was die Aussagefähigkeit der Ergebnisse angesichts eher geringer Fallzahlen stark beeinträchtigen kann.

Dies zeigt, dass die ex-post-Strategie einer statistischen Selektionskorrektur mitunter nur ein schlechter Ersatz für die ex-ante-Strategie einer zufälligen Programmzuweisung ist. Der Beitrag vermittelt einen Eindruck von der Größenordnung, die dabei zutage treten kann und unterstreicht damit die Notwendigkeit zur Durchführung von Modellprojekten in Form von sozialen Experimenten. Soziale Experimente liefern im Gegensatz zu aufwändigen ökonometrischen Modellen selbst bei geringen Fallzahlen einfache und glaubwürdige Schätzungen von Programmeffekten und hängen nicht von statistisch nicht überprüfbaren Annahmen ab (Ashenfelter 1987). Die Durchführung eines Modellprojekts als soziales Experiment erfordert dabei nicht unbedingt eine strikte Zuweisung im Rahmen eines Zufallsverfahrens. In der Praxis können beispielsweise auch Kapazitätsbeschränkungen als 
quasi-zufälliger Zuweisungsmechanismus genutzt werden. Allerdings dürfen dabei keine Ermessensspielräume zum Tragen kommen.

\section{Workfare: Eine Strategie zur Stärkung der Erwerbsanreize für gering Qualifizierte}

Für die hohe Arbeitslosigkeit von gering Qualifizierten werden häufig zu geringe Erwerbsanreize für Personen geltend gemacht, die am Markt nur einen niedrigen Stundenlohn erzielen können (siehe bspw. Christofides 2000 oder Schneider und Uhlendorff 2005). Wenn die resultierenden Vollzeiteinkommen nicht hinreichend weit über dem Einkommensniveau liegen, das durch Transferansprüche erzielt werden kann, lohnt sich die Ausübung einer entsprechenden Tätigkeit kaum. Die staatlich garantierte Mindestsicherung erzeugt auf diese Weise einen impliziten Mindestlohn.

Bislang hat die Politik vor allem mit Hilfe von Qualifizierungsmaßnahmen und Lohnsubventionen versucht, dem Problem zu begegnen. Der Erwerb von zusätzlichen Qualifikationen ermöglicht den Zugang zu besser entlohnten Tätigkeiten und erleichtert damit auf indirektem Wege die Überwindung des impliziten Mindestlohns. Mit Hilfe von Kombilöhnen kann das gleiche Ziel auf direktem Wege erreicht werden. Beide Strategien stoßen jedoch an ihre Grenzen. So ist die Effektivität von Qualifizierungsmaßnahmen umstritten (siehe bspw. Caliendo und Steiner 2005 oder Martin und Grubb 2001) und Lohnsubventionen unterliegen der Gefahr von Mitnahmeeffekten, deren Kosten den Nutzen einer Erwerbsaufnahme bei weitem übersteigen können (siehe bspw. Schneider et al. 2002b oder Bonin et al. 2003).

Workfare ist demgegenüber eine Option, die auf eine Absenkung des impliziten Mindestlohns abzielt, ohne dabei das Niveau der Grundsicherung in Frage stellen zu müssen (Bonin et al. 2007). Dies wird durch eine Kopplung des Grundsicherungsanspruchs an eine Pflicht zur Gegenleistung erreicht. Die Gegenleistung kann in Form von Arbeit erbracht werden, aber auch in Form von Bewerbungsaktivitäten oder einer Qualifizierungsmaßnahme. Die Pflicht zur Gegenleistung bewirkt, dass ein breiteres Spektrum von niedrig entlohnten Tätigkeiten am Markt akzeptabel wird, weil beim Vergleich zwischen Transfereinkommen und Erwerbseinkommen nur noch die Höhe des erzielbaren Einkommens ausschlaggebend ist.

Die jüngeren Wurzeln des Workfare-Konzepts finden sich vor allem in den USA. Unter dem Motto „Making Work Pay“ wurde dort in den 1970er Jahren der Earned Income Tax Credit (EITC) eingeführt (Blank 2002). Bei dem EITC handelt es sich zwar auf den ersten Blick um eine Lohnsubvention, doch im Kontext eines ansonsten praktisch fehlenden sozialen 
Sicherungssystems wurde de facto eine Situation geschaffen, in der staatliche Leistungen der Grundsicherung nur erhält, wer gleichzeitig einer Erwerbstätigkeit nachgeht. Der Begriff Workfare entstand erst im Zuge einer weiteren Sozialstaatsreform in den USA in den 1990er Jahren. Der 1996 eingeführte Welfare Reform Act wurde durch das Motto „from welfare to work“ geprägt. Daraus entwickelte sich dann der Kunstbegriff Workfare.

Die positiven Erfahrungen in den USA waren der Anlass, das Workfare-Konzept auch in Deutschland zu erproben. Unter dem Stichwort Aktivierung fand die Workfare-Idee im Rahmen des Modellprojekts MoZArT² ihren Niederschlag, an dem von 2001-2004 mehrere Großkommunen teilnahmen (Hess et al. 2004). In diesem Kontext ist auch das Projekt „Arbeit Sofort!“ zu sehen. Nicht zuletzt wurde mit der Schaffung von sogenannten „Ein-Euro-Jobs“ im Zuge der Hartz-Reform ein wesentliches Element des Workfare-Prinzips in die Sozialgesetzgebung aufgenommen.

Bislang existieren nur wenig gesicherte Erkenntnisse über die Wirkung von WorkfareAnsätzen im Kontext des deutschen Steuer- und Transfersystems. Simulationsrechnungen auf der Basis von Arbeitsangebotsschätzungen gehen davon aus, dass durch eine Einführung von Workfare bis zu 1,4 Mio. arbeitslose Bezieher von Leistungen der Grundsicherung eine Erwerbstätigkeit anstreben würden (Bonin et al. 2007). Demgegenüber kommen Bender et al. (2008) im Rahmen einer empirischen Untersuchung zu dem Schluss, dass die Reservationslöhne von Beziehern von Arbeitslosengeld II trotz einer Stärkung des Prinzips des Förderns und Forderns im Durchschnitt weitgehend stabil auf einem vergleichsweise hohen Niveau verharren. Offen bleibt dabei jedoch, inwieweit sich die konkrete Konfrontation mit Zwangsmaßnahmen wie beispielsweise einem Ein-Euro-Job auf den Reservationslohn auswirkt. Dessenungeachtet zeigt sich, dass die Einschränkung der Bezugsmöglichkeiten für Lohnersatzleistungen im Zuge der Hartz-Reform eine antizipative Wirkung entfaltet hat. So sind die Akzeptanzlöhne von Beziehern von Versicherungsleistungen (Arbeitslosengeld I) im Zuge der Hartz-Reform spürbar gesunken (Schneider 2008). In der Folge ist nicht nur die durchschnittliche Dauer der Arbeitslosigkeit, sondern auch der Zustrom in die Langzeitarbeitslosigkeit deutlich zurück gegangen.

\section{Die Umsetzung von Workfare im Rahmen von „Arbeit Sofort!“}

Das Projekt „Arbeit Sofort!“ wurde in der Zeit vom 01.09.2002 bis zum 30.08.2003 im Berliner Bezirk Charlottenburg-Wilmersdorf durchgeführt. Es galt zu dieser Zeit als

2 Modellvorhaben zur Verbesserung der Zusammenarbeit von Arbeitsämtern und Trägern der Sozialhilfe. 
neuartiges Programm zur Integration von Sozialhilfebeziehern in das Erwerbsleben. Zielgruppe waren erwerbsfähige Sozialhilfeantragsteller zwischen 18 und 25 Jahren. Die innovativen Elemente des Programms bestanden in der unmittelbaren Zuweisung von Neuantragstellern in das Projekt sowie in dem Workfare-Charakter. Die Antragsteller erhielten nur dann Unterstützungszahlungen, wenn sie an dem Projekt teilnahmen. Das Programm bestand aus Qualifizierungs- und Beschäftigungsmodulen, wobei Projektinhalt und -ablauf auf den jeweiligen Teilnehmer abgestimmt wurden. Mit dem Projekt „Arbeit sofort“ wurde das Ziel verfolgt, die Teilnehmer in den ersten Arbeitsmarkt zu integrieren und somit die Hilfebedürftigkeit zu überwinden und so genannten Sozialhilfekarrieren vorzubeugen. ${ }^{3}$

Das Projekt wurde gemeinsam vom Bezirksamt Charlottenburg-Wilmersdorf und dem Arbeitsamt getragen. Hierbei übernahm das Sozialamt die Kosten, die durch die Teilnehmerentlohnung sowie eventuelle Mietzuschüsse anfielen. Das Arbeitsamt trug die Programmkosten. Teilnehmer waren dazu verpflichtet, sich zu Beginn der Maßnahme beim Arbeitsamt als Arbeit suchend zu registrieren.

Bei dem Projektträger handelte es sich um einen Trägerverbund, zu dem sich drei Trägergesellschaften zusammengeschlossen hatten. Die einzelnen Trägergesellschaften waren bereits vor dem Projekt „Arbeit sofort“ mit Bildungs- und Beschäftigungsprojekten in Berlin tätig. Am Tag der Sozialhilfeantragstellung wurden die Teilnehmer direkt an den Projektträger vermittelt, am selben Tag fand bei dem Projektträger ein Erstgespräch statt. Es wurde ein Praktikantenvertrag zwischen dem Teilnehmer und dem Projektträger abgeschlossen. Dabei handelte sich um ein versicherungspflichtiges Beschäftigungsverhältnis, das auf maximal 6 Monate befristet war. Die Teilnehmer erhielten 434 Euro monatlich zuzüglich eventueller Mietkosten. Ihre Vergütung lag somit nur geringfügig über dem Sozialhilfeniveau. Die Vergütung konnte täglich ausbezahlt werden, so dass die Teilnehmer bereits am ersten Tag Geld erhalten konnten. Die Teilnahme an „Arbeit Sofort!“ war verpflichtend, d.h. Personen die nicht an der Maßnahme teilnahmen, obwohl sie zugewiesen worden waren, erhielten keine Sozialhilfe.

Zu Beginn der Maßnahme durchliefen die Teilnehmer ein mehrtägiges Profiling. Innerhalb dieses Profilings wurde für jeden Teilnehmer ein Leistungsprofil erstellt. Dieses Leistungsprofil beinhaltete bspw. bisherige Arbeitserfahrung, individuelle Interessen, Sachverhalte, die eine Arbeitsaufnahme verhinderten wie bspw. eine mögliche Verschuldung, den eventuellen Qualifizierungsbedarf zur Erlangung einer zukünftigen Beschäftigung, sowie

3 Die rechtliche Grundlage des Projekts bildeten die Paragraphen 18, 19 und 20 des

Bundessozialhilfegesetzes (BSHG), in denen die so genannte Hilfe zur Arbeit geregelt wurde. 
eine generelle Beurteilung der Beschäftigungsfähigkeit. Zudem wurde ein Bewerbertraining durchgeführt sowie grundlegende Fähigkeiten in Deutsch und Mathematik und das technische Verständnis erhoben. Aufbauend auf diesem Profiling wurden individuelle Handlungspläne mit dem Ziel der Integration in den ersten Arbeitsmarkt erstellt. Erst im Anschluss an das Profiling durchliefen die Teilnehmer die eigentlichen Qualifizierungs- und Beschäftigungsmodule von „Arbeit Sofort!“.

Zugelassen waren Personen, deren Sozialhilfeanspruch mindestens 207,79 Euro betrug. Diese Grenze ergab sich aus einer Abwägung von erwarteten Kosten und Nutzen des Programms seitens der Sozialbehörde. Von der Programmteilnahme ausgeschlossen waren Personen, die eine klare berufliche oder ausbildungsbezogene Zukunftsperspektive hatten und die sich daher nur vorübergehend im Sozialhilfebezug befanden.

Die Teilnehmerzahl war auf maximal 100 Personen zur gleichen Zeit beschränkt. Dies ist insofern von Bedeutung, als es in methodischer Hinsicht die Chance geboten hätte, Kapazitätsbeschränkungen als quasi-zufälligen Programmzuweisungsprozess zu nutzen. Da der Zeitpunkt der Bedürftigkeitsmeldung als Zufallsereignis interpretierbar ist, eignet sich der Kreis der aufgrund von Kapazitätsbeschränkungen abgelehnten Programmteilnehmer als Vergleichsgruppe für die Teilnehmer. Leider wurde bei der Durchführung des Modellprojekts die Chance vertan, diesen Mechanismus entsprechend zu nutzen. Stattdessen wurde von den Sachbearbeitern von der Möglichkeit Gebrauch gemacht, Neuantragsteller zu einem späteren Zeitpunkt in das Programm einzuweisen, wenn ihnen die jeweilige Person dafür geeignet erschien. Ebenso konnten Sachbearbeiter entscheiden, einen Neuantragsteller trotz freier Programmplätze nicht zu dem Programm zuzulassen, wenn ihnen die jeweilige Person trotz Erfüllung der formalen Kriterien ungeeignet erschien. Aus diesem Grund sind entsprechende Selektionskorrekturverfahren beim Vergleich von Programmteilnehmern und aus Kapazitätsgründen abgelehnten Sozialhilfeempfängern unerlässlich.

Insgesamt haben an dem Projekt 279 Personen teilgenommen. Allerdings gehen nur etwa zwei Drittel davon in die anschließende Evaluation ein. Der Grund dafür besteht darin, dass zu Beginn des Projekts aus kameralistischen Erwägungen heraus von dem Prinzip abgewichen wurde, ausschließlich Neuantragsteller in das Programm aufzunehmen. Da das Programm erst im Herbst des Jahres 2002 starten konnte, bestand die Gefahr, dass die bereitgestellten Mittel bis zum Ende des Haushaltsjahres nicht hätten ausgeschöpft werden können, wenn ausschließlich Neuantragsteller aufgenommen worden wären. Deshalb entschloss sich das Sozialamt dazu, zum Programmstart auch Personen aus dem Bestand der Sozialhilfebezieher 
in das Programm einzubeziehen. Die Kriterien für die Auswahl dieser Personengruppe wurden mehr oder weniger ad-hoc festgelegt. So berichteten die zuständigen Sachbearbeiter, dass sie gezielt auf Personen zugegangen seien, bei denen zu erwarten war, dass sie freiwillig an dem Programm teilnehmen würden. Da für diesen Personenkreis von vorneherein keine geeignete Vergleichsgruppe definierbar ist, bleiben diese Personen von der weiteren Analyse ausgeschlossen.

\section{Datengrundlage}

Ausgangspunkt der verwendeten Datenbasis sind Informationen über Zugänge in Sozialhilfe im Bezirk Charlottenburg-Wilmersdorf in den Jahren 2002 und 2003. Neben Informationen über den Zugang in „Arbeit Sofort!“ werden im Sozialamt nur Basisdaten wie Alter, Geschlecht, etc. erfasst, die zur Prüfung der Bedürftigkeit erforderlich sind. Informationen über Beschäftigungsphasen vor dem Sozialhilfebezug sind dort ebensowenig vorhanden wie Informationen über Beschäftigungsphasen im Anschluss an den Sozialhilfebezug. Übergänge in Beschäftigung als wesentliches Erfolgskriterium lassen sich mit diesen Daten folglich nicht überprüfen. Zwar wäre es möglich gewesen, entsprechende Daten mittels einer Befragung zu erheben, doch ist dies bei der vorliegenden Zielgruppe erfahrungsgemäß mit einer sehr geringen Ausschöpfungsquote verbunden (vgl. bspw. Schneider et al. 2002a). Somit schied diese Option angesichts der niedrigen Fallzahlen von vorneherein aus. Stattdessen wurde von der Möglichkeit Gebrauch gemacht, die erforderlichen individuellen Erwerbsverlaufsinformationen aus den Geschäftsdaten der Bundesagentur für Arbeit zu beziehen und mit den Sozialamtsdaten zu koppeln. Für diesen Zweck stehen die so genannten Integrierten Erwerbsbiografien (IEB) zur Verfügung (Jacobebbinghaus und Seth, 2007). Die IEB enthalten exakte Informationen zu Beschäftigungs- und Arbeitslosigkeitsphasen sowie den daraus generierten Einkommen.

Eine besondere Herausforderung bestand darin, die Datensätze des Sozialamtes und der Bundesagentur zusammenzuführen. Es liegt keine eindeutige Personenkennzeichnung bspw. in Form der Sozialversicherungsnummer vor, die von beiden Institutionen verwandt wird. Deswegen musste der Abgleich über geeignete Personeninformationen vorgenommen werden. Aufgrund der besonderen datenschutzrechtlichen Problematik wurde dieser Abgleich treuhänderisch im Forschungsdatenzentrum der Bundesagentur für Arbeit im Institut für Arbeitsmarkt- und Berufsforschung (FDZ BA/IAB) vorgenommen. Mit Hilfe eines Pseudonyms - d.h. einer willkürlich vergebenen, aber eindeutigen Personennummer - wurde 
anschließend ein kombinierter Datensatz erstellt. Im Einzelnen umfasste das Vorgehen die nachfolgend beschriebenen Schritte (vgl. Abbildung 1).

Im Sozialamt wurde zunächst ein Datensatz erstellt, der Teilnehmer an „Arbeit sofort“ bzw. für einen Vergleich geeignete Nichtteilnehmer umfasste. Bei den für einen Vergleich geeigneten Nichtteilnehmern handelt es sich um Personen, die zwischen September 2002 und August 2003 einen Antrag auf Sozialhilfe gestellt haben und deren Sozialhilfeanspruch mindestens 207,79 € betrug. ${ }^{4}$ Diese Gruppe umfasst insgesamt 928 Personen, die potenziell für den Vergleich mit der Gruppe der 189 Programmteilnehmer zur Verfügung stehen. Darin enthalten sind allerdings auch diejenigen Personen, die deshalb nicht in das Programm zugewiesen wurden, weil sie bereits eine Stelle in Aussicht hatten. Da eine entsprechende Information in den Sozialamtsdaten nicht festgehalten wurde, lassen sich diese Personen nicht identifizieren. Der einfache Vergleich von Teilnehmern mit Nichtteilnehmern an „Arbeit Sofort!“ ist insofern von vorneherein zu Lasten der Teilnehmer verzerrt.

Die Daten wurden mit einem Pseudonym versehen und den beteiligten Forschungsinsituten (DIW Berlin und IZA) in anonymisierter Form zur Verfügung gestellt. Zusätzlich wurde vom Sozialamt ein Hilfsdatensatz erstellt, der lediglich den Namen, das Geburtsdatum, die Postleitzahl und das Pseudonym enthielt. Dieser wurde an das FDZ BA/IAB übermittelt und dort in einem mehrstufigen Verfahren mit den IEB möglichst eindeutig abgeglichen. Zur maximalen Ausschöpfung wurde beim Abgleich unter anderem die Namensinformation variiert und in weiteren Iterationen nur der jeweils erste Teil des Namens bzw. nur der Nachname abgeglichen.

Für mehr als 95\% der Teilnehmer konnte im FDZ BA/IAB mindestens ein Konto identifiziert werden. Für $80 \%$ wurde ein, für 14\% wurden zwei und für 1\% wurden mehr als zwei Konten ermittelt. Von den Personen aus der Kontrollgruppe konnte mindestens für 87\% mindestens ein Konto identifiziert werden. Für 78\% wurde ein, für 8\% wurden zwei und für 1\% mehr als 2 Konten ermittelt. Darüber hinaus kam es vor, dass ein Konto mehreren Sozialhilfefällen zugeordnet werden konnte. Dies kann bspw. dadurch entstehen, dass mehrere Personen mit gleichem Nachnamen in einem Haushalt leben.

Anschließend wurde vom FDZ BA/IAB ein Datensatz erstellt, der neben dem Pseudonym Informationen zur Erwerbshistorie aus den IEB-Daten enthält. Dieser Datensatz wurde an die an dem Evaluationsprojekt beteiligten Institute (DIW Berlin und IZA) übermittelt. Dort

$4 \quad$ Nach Berechnungen des Sozialamts lohnte sich für den Bezirk eine Zuweisung von Sozialhilfeberechtigten in „Arbeit Sofort!“ nicht, wenn deren Sozialhilfeanspruch unterhalb dieses Betrags lag. 
wurden abschließend die Daten des Sozialamts über das gemeinsame Pseudonym mit den IEB-Daten verknüpft.

Abbildung 1: Zusammenfügung der administrativen Datensätze

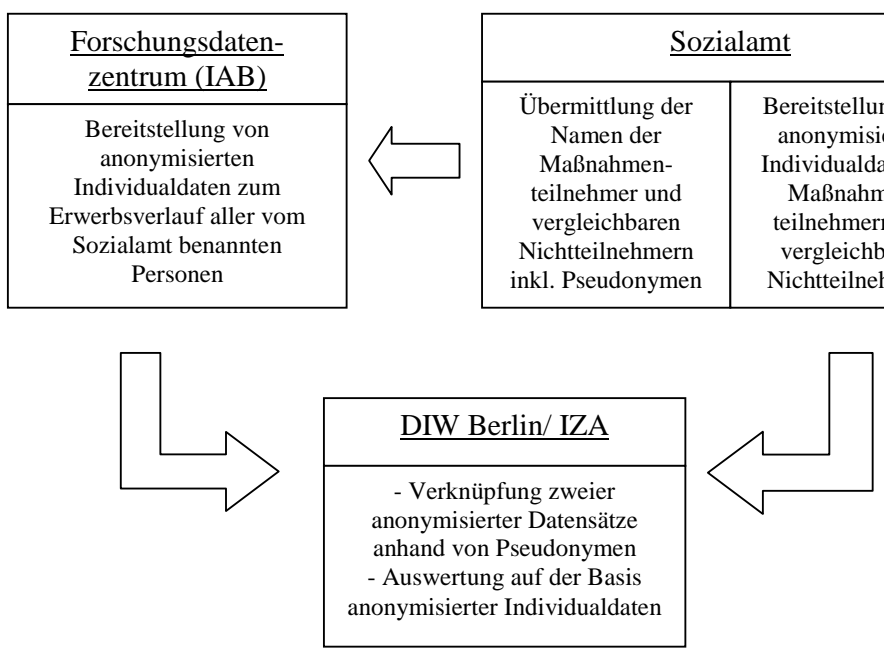

\section{Datenbereinigung}

Vor Beginn der Datenanalyse mussten mehrdeutige Zuordnungen durch

Plausibilitätsprüfungen ermittelt und aus dem Datensatz ausgeschlossen werden. Dabei kam folgende Heuristik zur Anwendung:

Eine offensichtlich fehlerhafte Zuordnung von BA-Konten besteht bei Unterschieden in den Angaben über das Geschlecht und/oder das Alter einer Person im Vergleich zu den Angaben des Sozialamtes. Weichen Geschlecht und/oder Alter um mehr als zwei Jahre voneinander ab, gehen wir von einer fehlerhaften Zuordnung eines BA-Kontos aus.

Weitere Hinweise auf fehlerhafte Zuordnungen ergeben sich durch Informationen in den BA-Daten zu Einkommen und Beschäftigung während des Bezugs von Sozialhilfe. Wenn eine Person im Sozialhilfebezug steht, wird ihr Einkommen relativ niedrig sein. IEBKonten, die für diesen Zeitraum einen relativ hohen Verdienst (Tagesentgelt > 30€) ausweisen, sind damit höchstwahrscheinlich falsch zugeordnet.

Ein ähnliches Kriterium ergibt sich bei Betrachtung des Erwerbsverlaufes vor Eintritt in Sozialhilfe. IEB-Konten, die für die Quartale vor Sozialhilfeeintritt einer Person eine durchgehende Beschäftigung bei relativ hohem Einkommen (Tagesentgelt> 30€) ausweisen, dürften ebenfalls falsch zugeordnet sein, da diese Person bei Arbeitslosigkeit Anspruch auf Arbeitslosengeld hätte und damit nicht direkt in Sozialhilfe eintreten würde. 


\section{Informationen in den Datensätzen}

Neben basisdemographischen Informationen enthalten die Sozialhilfedaten Informationen zur Haushaltssituation, sowie zu Religionszugehörigkeit und eventuellen Behinderungen. Füllgrad und Zuverlässigkeit der Informationen schwanken jedoch. Verlässlich sind vor allem die Informationen mit einem Bezug zur Auszahlung von Sozialhilfe. ${ }^{5}$ Leider können Personen, die für die Teilnahme an „Arbeit Sofort!“ ausgewählt wurden, dann aber nicht zum Erstgespräch beim Projektträger erschienen, nicht identifiziert werden, da eine entsprechende Erfassung in den Sozialamtsdaten nicht vorgesehen war. Ein möglicher Abschreckungseffekt des Programms lässt sich daher nicht ermitteln.

Die IEB enthalten neben sozioökonomischen Informationen wie Alter und Bildung detaillierte Angaben zu sozialversicherungspflichtiger Beschäftigung und dem daraus resultierendem Einkommen, dem Bezug von Arbeitslosengeld sowie der Teilnahme an Maßnahmen der aktiven Arbeitsmarktpolitik.

Beide Datensätze wurden für die Ermittlung von Eigenschaften genutzt, die die Teilnehmer und Nichtteilnehmer vor dem Eintritt in die Maßnahme aufwiesen. Mit Hilfe der Daten der IEB wurden Informationen über den Erwerbsverlauf nach Eintritt in die Maßnahme (Teilnehmer) bzw. nach Eintritt in die Sozialhilfe (Nichtteilnehmer) generiert. Im Fokus steht dabei die Zielvariable „Ungeförderte Beschäftigung“. Ungeförderte Beschäftigung ist definiert als Phase sozialversicherungspflichtiger Beschäftigung, während der die Teilnehmer keine Leistungen von der Arbeitsagentur bezogen haben, nicht als arbeitslos gemeldet waren und an keiner arbeitsmarktpolitischen Maßnahme wie bspw. Förderung durch einen Lohnkostenzuschuss oder Weiterbildungsmaßnahmen teilgenommen haben. Informationen zu sozialversicherungspflichtiger Beschäftigung liegen mit einem hinreichenden Füllgrad bis Ende 2004 vor. Die Zielvariable wurde ab dem Programm- bzw. Sozialhilfeeintritt in zweiwöchigen Intervallen gebildet und liegt für einen Zeitraum von bis zu 12 Monaten nach Programmeintritt vor. ${ }^{6}$ Bei einer Programmlaufzeit von bis zu 6 Monaten beobachten wir die

Ergebnisvariable für jeden Teilnehmer somit für mindestens 6 Monate nach Austritt aus dem Programm „Arbeit sofort“.

5 Dies wurde mehrfach von den Mitarbeitern des Sozialamts betont und ist auch in den Daten erkennbar. So enthält die Variable Religion lediglich bei ca. 2\% der Fälle einen gültigen Wert. Die Beobachtungsperiode von bis zu 12 Monaten ergibt sich dadurch, dass für einige zusätzliche Untersuchungen auch Personen zum Vergleich herangezogen wurden, die nach Beendigung des Projekts in die Sozialhilfe eingetreten sind (vom 15.07. bis zum 31.12.2003). Die Ergebnisse dieser zusätzlichen Untersuchungen weichen qualitativ nicht von den hier berichteten ab. 


\section{Ergebnisse}

Der Effekt einer arbeitsmarktpolitischen Maßnahme lässt sich statistisch durch einen Vergleich zwischen einer Gruppe von Teilnehmern an dieser Maßnahme und einer Gruppe von Personen, die an dieser Maßnahme nicht teilgenommen haben, ermitteln. Dies setzt voraus, dass sich Teilnehmer und Nichtteilnehmer außer im Hinblick auf die Maßnahmenteilnahme nicht systematisch voneinander unterscheiden. Bezogen auf ein bestimmtes Evaluationskriterium - hier dem Beschäftigungsstatus - resultiert der Maßnahmeneffekt aus der Differenz zwischen dem Wert, der sich mit Maßnahmenteilnahme und dem Wert, der sich ohne Maßnahmenteilnahme ergibt.

Die genannte Voraussetzung ist dann erfüllt, wenn die Zuordnung in Teilnehmer- und Kontrollgruppe zufällig erfolgt ist. In diesem Fall liefert ein einfacher Vergleich von Teilnehmern und Nichtteilnehmern einen unverzerrten Maßnahmeneffekt. Im vorliegenden Fall ist jedoch davon auszugehen, dass der Zugang in die Maßnahme nicht nach einem reinen Zufallsprinzip erfolgt ist. Dies unterstreicht ein deskriptiver Vergleich einiger ausgewählter beobachtbarer Eigenschaften für Teilnehmer und Nichtteilnehmer. So sind die Teilnehmer im Durchschnitt jünger, sie sind eher männlich, und befanden sich vor Eintritt in die Sozialhilfe zu einem deutlich geringeren Anteil in Beschäftigung.

[Tabelle 1 ungefähr hier]

Ein einfacher Vergleich beider Gruppen deutet zunächst auf einen negativen Effekt der Programmteilnahme hin: Die Teilnehmer weisen mit einem Anteil von 10,5 \% einen um ca. 1.8 Prozentpunkte geringeren Anteil aus als die Nichtteilnehmer mit 12,3 \%. ${ }^{7}$ Angesichts der bereits erwähnten Tatsache, dass die Gruppe der Nichtteilnehmer gezielt auch die Personen umfasst, die bereits eine Beschäftigung in Aussicht hatten, gestattet dieser Befund keine inhaltlichen Schlussfolgerungen. Es ist vielmehr notwendig, die a-priori-Unterschiede zwischen Teilnehmern und Nichtteilnehmern hinsichtlich der Wahrscheinlichkeit für die Aufnahme einer Beschäftigung in geeigneter Form zu berücksichtigen. Im günstigsten Fall lassen sich diese durch beobachtete Merkmale wie etwa die vorhergehende Beschäftigungshistorie modellieren.

Hierfür werden zunächst einfache Probit-Modelle geschätzt. Die abhängige Variable ist die individuelle Beschäftigungswahrscheinlichkeit. Diese Wahrscheinlichkeit wird als

Bei Personen, für die keine Information über ihren Erwerbsstatus vor und nach Eintritt in Sozialhilfe in den Daten der Bundesagentur für Arbeit aufgefunden wurde, nehmen wir an, dass sie nicht erwerbstätig waren und aus diesem Grund keine Informationen in den Daten vorhanden waren. 
Linearkombination mehrerer unabhängiger Variablen modelliert. Der Koeffizient für den Teilnahme-Indikator $D$ spiegelt den kausalen Effekt der Teilnahme wider. Tabelle 2 gibt die entsprechenden marginalen Effekte wider.

[Tabelle 2 ungefähr hier]

Der Punktschätzer deutet im Gegensatz zum einfachen Vergleich der Ergebnisvariablen darauf hin, dass die Teilnahme an „Arbeit Sofort!“ die Beschäftigungswahrscheinlichkeit erhöht. Allerdings ist der Effekt mit 2,1 Prozentpunkten nicht sehr groß und statistisch nicht signifikant.

Zusätzlich zeigt sich, dass Männer eine signifikant höhere Beschäftigungswahrscheinlichkeit aufweisen als Frauen und dass die Beschäftigungswahrscheinlichkeit positiv mit vorheriger Beschäftigung und einer Berufsausbildung korreliert ist, während Verheiratete 12 Monate nach Eintritt in die Sozialhilfe zu einem geringeren Anteil sozialversicherungspflichtig beschäftigt sind.

Das geschilderte Probit-Modell beruht auf spezifischen parametrischen Annahmen. Dazu zählt vor allem, dass der Modellzusammenhang auch für Wertebereiche der erklärenden Merkmale gilt, für die es keine beobachtete Entsprechung zwischen Teilnehmern und Nichtteilnehmern gibt. Dies hat zur Folge, dass das Ergebnis in irreführender Weise von der Verteilung der erklärenden Merkmale beeinflusst werden kann. Im Gegensatz dazu werden beim sogenannten Matching-Verfahren keine parametrischen Annahmen getroffen. Stattdessen wird durch die Bildung von statistischen Zwillingen dafür gesorgt, dass der Vergleich der Teilnehmer nur mit Nichtteilnehmern erfolgt, die den Teilnehmern möglichst ähnlich sind und dass darüber hinaus nur Teilnehmer in den Vergleich eingehen, für die sich vergleichbare Nichtteilnehmer finden lassen. Der Preis für die Anwendung dieses Verfahrens besteht in einer Reduktion der Fallzahl. Statt 1.117 Beobachtungen wie im Probit-Modell stehen bei Anwendung des Matching-Verfahrens nur 396 Beobachtungen zur Verfügung.

Wir wenden ein Propensity-Score Matching an, wobei in einem ersten Schritt die Wahrscheinlichkeit geschätzt wird, an der Maßnahme teilzunehmen. In einem zweiten Schritt werden die Teilnehmer und die Nichtteilnehmer auf Grundlage der Teilnahmewahrscheinlichkeit einander zugeordnet. Der kausale Effekt der Teilnahme ergibt sich anschließend durch einen einfachen Vergleich beider Gruppen miteinander ${ }^{8}$.

$8 \quad$ Für eine gute Übersicht über die Anwendung von Matching-Verfahren siehe Caliendo und Kopeinig 2008. 
Vor dem Matching bestehen viele signifikante Unterschiede zwischen Teilnehmern und Nichtteilnehmern. Mit Hilfe des Matchings werden die Teilnehmer und Nichtteilnehmer in Hinblick auf ihre beobachtbaren Unterschiede angeglichen. Die Reduktion der Unterschiede ist zufriedenstellend, siehe Tabelle A1 im Anhang. In Tabelle 3 werden die Ergebnisse des Matching-Ansatzes berichtet. Auch hier ergibt sich ein positiver Effekt der Teilnahme an „Arbeit Sofort!“. Der Punktschätzer beträgt 3.5 Prozentpunkte, ist aber nach wie vor nicht signifikant von 0 verschieden.

[Tabelle 3 ungefähr hier]

Sowohl das Probit-Modell als auch das Matching-Modell beruhen auf der Annahme, dass sich Selektionseffekte ausschließlich an beobachteten Merkmalen festmachen lassen. Diese Annahme lässt sich empirisch zwangsläufig nicht überprüfen. Die Berichte der Sachbearbeiter sprechen jedoch dafür, dass für die Maßnahmenzuweisung auch Kriterien eine Rolle spielen, die in den verfügbaren Daten nicht abgebildet sind (Alkoholismus, psychische Probleme, etc.). Zudem ist nicht sicher, ob die a-priori-Beschäftigungsaussichten, die zum Ausschluss von der Programmteilnahme führten, durch beobachtbare Merkmale hinreichend kontrolliert werden können.

Um potenzielle Unterschiede in nichtbeobachtbaren Merkmalen kontrollieren zu können, nutzen wir die Tatsache aus, dass auf der einen Seite die Wahrscheinlichkeit der Maßnahmenteilnahme von der Programmbelegung abhängt, man aber auf der anderen Seite davon ausgehen kann, dass die Programmbelegung keinen kausalen Einfluss auf die späteren Erwerbschancen hat. Ist die Programmbelegung hoch, so ist die Wahrscheinlichkeit der Teilnahme relativ gering im Vergleich zur Situation mit geringer Programmbelegung. Bei geringer Programmbelegung treten auch Personen in das Programm ein, die aufgrund beobachtbarer und unbeobachtbarer Eigenschaften bei hoher Auslastung nicht eingetreten wären. Dieser Effekt erlaubt daher die simultane Modellierung des Zugangsprozesses in die Maßnahme und der Erwerbswahrscheinlichkeit mit Hilfe eines bivariaten Probit Modells.

Dieser Ansatz ermöglicht es, für eine potenzielle Selektion auf Grundlage von unbeobachteten Eigenschaften zu kontrollieren. Zwar kann hierfür wieder die volle Fallzahl genutzt werden, doch ergeben sich dafür erhöhte statistische Unsicherheiten aufgrund der komplexeren Modellstruktur.

Die Selektionsgleichung, mit der die Wahrscheinlichkeit des Eintritts in das Programm modelliert wird, ist in Anhang in Tabelle A2 dokumentiert. Die Auslastung des Programms, 
gemessen an der Teilnehmerzahl zum jeweiligen Zeitpunkt des Eintritts in die Sozialhilfe, hat einen signifikant negativen Einfluss auf die Teilnahmewahrscheinlichkeit.

[Tabelle 4 ungefähr hier]

In Tabelle 4 sind die entsprechenden Effekte der Variablen auf die Beschäftigungswahrscheinlichkeit 12 Monate nach Eintritt in die Maßnahme aufgeführt. Auch in diesem Modell ist der gemessene Effekt der Teilnahme auf die Erwerbswahrscheinlichkeit nicht signifikant von 0 verschieden. Allerdings fällt der marginale Effekt sehr deutlich aus. Teilnehmer an „Arbeit Sofort!“ weisen demnach 12 Monate nach Maßnahmebeginn eine um 27 Prozentpunkte höhere Beschäftigungswahrscheinlichkeit auf als Nichtteilnehmer. Diese ökonomisch sehr relevante Größenordnung spricht für die Wirksamkeit des Workfare-Konzepts. Dennoch ist das Ergebnis aufgrund einer zu geringen Fallzahl statistisch nicht belastbar. Vergleicht man den Standardfehler des Maßnahmeneffektes im einfachen Probit Modell mit dem Standardfehler im Modell mit simultaner Selektionskorrektur, so zeigt sich, dass der Standardfehler im bivariaten Probit Modell um den Faktor 4.5 höher liegt. Dies illustriert den Preis, der aus einem inkonsequenten Projektdesign resultiert. Mit Hilfe statistischer Verfahren lassen sich Selektionsverzerrungen im Nachhinein zwar korrigieren, aber das bedeutet nicht unbedingt, dass damit für praktische Handlungsempfehlungen viel gewonnen wird.

\section{Schlussbemerkungen}

Die vorliegende Untersuchung geht der Frage nach, ob die Anwendung des WorkfarePrinzips dazu geeignet ist, die Anreizprobleme der Grundsicherung zu überwinden. Das Ergebnis spricht dafür, auch wenn die üblichen statistischen Signifikanzkriterien auf der Grundlage der verwendeten Daten verfehlt werden. Überdies bestätigt sich einmal mehr, dass Selektionseffekte bei der Programmteilnahme zu irreführenden Schlussfolgerungen verleiten können, wenn sie nicht adäquat berücksichtigt werden.

Um Selektionseffekte auszuschließen, müssen entweder im Vorhinein entsprechende Vorkehrungen bei der Programmdurchführung getroffen werden, oder aber es muss im Nachhinein mit Hilfe statistischer Korrekturverfahren für ihre Neutralisierung gesorgt werden. Letzteres erfordert jedoch beträchtlich höhere Fallzahlen als sie im Rahmen von Modellprojekten üblicherweise zur Verfügung stehen.

Um eine höchstmögliche Fallzahlausbeute zu erhalten, wurde eine Verknüpfung von Sozialamtsdaten mit Erwerbsbiographie-Daten der Bundesagentur für Arbeit vorgenommen. 
Die Studie zeigt, dass eine solche Verknüpfung grundsätzlich eine effiziente Möglichkeit darstellt, den Erfolg von Modellprojekten in der Grundsicherung zu untersuchen. Für mehr als 90\% der untersuchten Fälle ließ sich ein erfolgreicher Datenabgleich herbeiführen. Der Nachteil einer Beschränkung auf Verwaltungsdaten besteht zwar darin, dass die Umstände, unter denen eine Beschäftigung gefunden wird oder auch nicht gefunden wird, im Dunkeln bleiben. Ohne die durchgeführte Verknüpfung wäre die Studie jedoch gar nicht durchführbar gewesen, denn der zu erwartende Rücklauf bei einer Befragung wäre erfahrungsgemäß erheblich niedriger ausgefallen.

Dass die Fallzahl dennoch nicht ausreicht, um statistisch signifikante Ergebnisse zu erhalten, resultiert aus einem Projektdesign, das unbeobachteten Selektionseffekte unterliegt. Hier offenbart sich ein verbreitetes Dilemma von Modellprojekten: Die Praxis der Projektdurchführung lässt sich häufig nicht mit den Anforderungen an eine belastbare Aussagefähigkeit der Ergebnisse in Einklang bringen. Die Gründe dafür sind zahlreich und nicht immer leicht zu überwinden. Mal sind es haushaltstechnische Gründe, die dazu zwingen, ein Programm mit Teilnehmern zu füllen, mal sind es fachliche Erwägungen der zuständigen Sachbearbeiter, die sich sowohl schwer damit tun, Probanden, die sie für die Teilnahme geeignet halten, zugunsten höherer Einsichten von der Teilnahme abzuhalten als auch damit, aus ihrer Sicht ungeeignete Kandidaten aus Gründen des Projektdesigns für die Teilnahme zuzulassen.

Darüber hinaus werden immer wieder vermeintlich moralische Argumente vorgebracht, wonach es nicht mit dem Gleichbehandlungsgrundsatz vereinbar sei, jemandem bewusst etwas vorzuenthalten, was jemand anderem bewusst gewährt werde. Dass es trotzdem auch anders geht, zeigen die erfolgreich durchgeführten Modellprojekte im Rahmen des bereits erwähnten MoZArT-Projekts, bei denen eine echte Zufallssteuerung bei der Programmzuweisung zum Einsatz kam. Leider bilden solche Beispiele in Deutschland bis heute eine seltene Ausnahmen in der Praxis von Modellprojekten. Im Übrigen ist nur schwer nachvollziehbar, wieso ein Prinzip, das in der Medizin allgemein anerkannt und als unverzichtbar angesehen wird, in der arbeitsmarktpolitischen Praxis nicht gelten soll.

Insofern ist die vorliegende Studie auch als ein Plädoyer dafür zu verstehen, dem Grundsatz eines erkenntnisorientierten Projektdesigns Vorrang vor kurzfristigen pragmatischen Erwägungen einzuräumen. Dies bedeutet die Vermeidung von unkontrollierten Selektionseffekten bei der Zuweisung in die Programmteilnahme. In der Praxis ist dies mitunter einfacher als oft angenommen, wie beispielsweise die Möglichkeit der Nutzung von 
Beschränkungen der Programmkapazität als Quasi-Zufallsprozess zeigt. Die Durchführung von Modellprojekten ohne Chance auf Erkenntnisgewinn stellt eine Vergeudung von Ressourcen dar. Die langfristigen Kosten des Nicht-Wissens dürften bedeutend höher sein als die kurzfristig auftretenden Kosten eines konsequent erkenntnisorientierten Projektdesigns. 


\section{Literatur}

Ashenfelter, O. (1987): The Case for Evaluating Training Programs with Randomized Trials, Economics of Education Review, 6, 333-338.

Bender, S., S. Koch, S. Meßmann und U. Walwei (2008): Was muten sich Arbeitslose zu? Lohnkonzessionen von ALG II-Empfängern, Sozialer Fortschritt, 57, 75-85.

Blank, R.M. (2002): Evaluating Welfare Reform in the United States, Journal of Economic Literature, 40, 1105-1166.

Blundell, R. und M. C. Dias (2009):Alternative Approaches to Evaluation in Empirical Microeconomics, Journal of Human Resources, 44, 565-640.

Bonin, H., A. Falk und H. Schneider (2007): Workfare - Praktikabel und gerecht, ifo Schnelldienst, 4/2007, 33-37

Bonin, H., W. Kempe und H. Schneider (2003): Kombilohn oder Workfare? Zur Wirksamkeit zweier arbeitsmarktpolitischer Strategien, Vierteljahrshefte zur Wirtschaftsforschung, 72, 51-67

Caliendo, M. und S. Kopeinig (2008): Some Practical Guidance for the Implementation of Propensity Score Matching, Journal of Economic Surveys, 22, 31-72.

Caliendo, M. und V. Steiner (2005): Aktive Arbeitsmarktpolitik in Deutschland: Bestandsaufnahme und Bewertung der mikroökonomischen Evaluationsergebnisse, Zeitschrift für Arbeitsmarktforschung, 38, 396-418.

Christofides, L. N. (2000): Social assistance and labour supply, Canadian Journal of Economics, 33, 715-741.

Hess, D. H. Schröder, M. Smid und C. Reis (2004): MoZArT - Neue Strukturen für Jobs Abschlussbericht der wissenschaftlichen Begleitforschung. Bundesministerium für Wirtschaft und Arbeit (Hrsg.), Dokumentation Nr. 541, Berlin.

Imbens, G. und J. Wooldridge (2008): Recent Developments in the Econometrics of Program Evaluation, Journal of Economic Literature, 47, 5-86.

Jacobebbinghaus, P. und S. Seth (2007): The German Integrated Employment Biographies Sample IEBS, Schmollers Jahrbuch, 127, 335-342.

Lee, M.-J. (2005): Micro-Econometrics For Policy, Program and Treatment Effects, Oxford University Press.

Martin, J.P. und D. Grubb (2001): What works and for whom: A review of OECD countries' experience with active labour market policies, Swedish Economic Policy Review, 8, 9-56.

Schneider, H. (2008): Die Veränderung der Lohnersatzleistungen und die Reform der Vermittlungsprozesse im SGB III, Vierteljahrshefte zur Wirtschaftsforschung, 77, 20-37

Schneider, H., C. Lang, M.T.W. Rosenfeld, W. Kempe und J. Kolb (2002a): Anreizwirkungen der Sozialhilfe auf das Arbeitsangebot im Niedriglohnbereich. Baden-Baden: Nomos Verlagsgesellschaft.

Schneider, H., K.F. Zimmermann, H. Bonin, K. Brenke, J. Haisken-DeNew und W. Kempe (2002b): Beschäftigungspotenziale einer dualen Förderstrategie im Niedriglohnbereich. IZA Research Report No. 5, Bonn.

Schneider, H. und A. Uhlendorff (2005): Transitions from Welfare to Employment: Does the Ratio between Labor Income and Social Assistance Matter? Schmollers Jahrbuch, 125, 51-61. 


\section{Tabellen}

\section{Tabelle 1: Deskriptiver Vergleich von Teilnehmern und Nichtteilnehmern}

\begin{tabular}{|c|c|c|}
\hline & Teilnehmer & Nichtteilnehmer \\
\hline $\begin{array}{l}\text { Ungeförderte Beschäftigung } 12 \text { Monate nach Eintritt } \\
\text { Alter }\end{array}$ & $\begin{array}{l}0,1053 \\
20,61 \\
(1,90)\end{array}$ & $\begin{array}{l}0,1226 \\
21,36 \\
(2,33)\end{array}$ \\
\hline Geschlecht (Mann=1) & 0,60 & 0,47 \\
\hline Verheiratet & 0,04 & 0,20 \\
\hline Stellung im Haushalt: HH Vorstand oder Ehepartner & 0,31 & 0,17 \\
\hline Stellung im Haushalt: Kind & 0,04 & 0,11 \\
\hline Stellung im Haushalt: Sonstiges & 0,65 & 0,72 \\
\hline Asylberechtigt & 0,01 & 0,05 \\
\hline Flüchtling & 0,01 & 0,01 \\
\hline EU-Ausländer & 0,09 & 0,07 \\
\hline Nicht-EU-Ausländer & 0,14 & 0,20 \\
\hline Kein Schulabschluss / Schulabschluss unbekannt & 0,38 & 0,33 \\
\hline Hauptschule & 0,37 & 0,24 \\
\hline Realschule & 0,20 & 0,32 \\
\hline Fachhochschulreife / Abitur & 0,05 & 0,11 \\
\hline Berufsausbildung & 0,06 & 0,18 \\
\hline $\begin{array}{l}\text { Prozentualer Anteil in Beschäftigung im 1. Quartal vor Eintritt } \\
\text { in Sozialhilfe }\end{array}$ & 0,02 & 0,07 \\
\hline $\begin{array}{l}\text { Prozentualer Anteil in Beschäftigung im 2. Quartal vor Eintritt } \\
\text { in Sozialhilfe }\end{array}$ & 0,03 & 0,11 \\
\hline $\begin{array}{l}\text { Prozentualer Anteil in Beschäftigung im 3. Quartal vor Eintritt } \\
\text { in Sozialhilfe }\end{array}$ & 0,04 & 0,12 \\
\hline $\begin{array}{l}\text { Prozentualer Anteil in Beschäftigung im 4. Quartal vor Eintritt } \\
\text { in Sozialhilfe }\end{array}$ & 0,06 & 0,13 \\
\hline
\end{tabular}

In Klammern unter den Mittelwerten sind die Standardabweichungen der Variablen angegeben, falls es sich nicht um Anteilswerte handelt. Teilnehmer: $\mathrm{n}=189$, Nichtteilnehmer: $\mathrm{n}=928$. 
Tabelle 2: Probit-Modell, Beschäftigungswahrscheinlichkeit 12 Monate nach Eintritt in das Programm

\begin{tabular}{|c|c|c|c|}
\hline Variable & Koeffizient & Robuster Standardfehler & Marginaler Effekt \\
\hline Teilnahme am Programm (D=1) & 0,117 & 0,139 & 0,0214 \\
\hline Alter & 0,022 & 0,026 & 0,0038 \\
\hline Geschlecht (Mann=1) & $0,259 * *$ & 0,103 & 0,0450 \\
\hline Verheiratet & $-0,314 * *$ & 0,153 & $-0,0476$ \\
\hline Stellung im Haushalt: HH Vorstand oder Ehepartner & $-0,008$ & 0,146 & $-0,0014$ \\
\hline Stellung im Haushalt: Kind & $-0,008$ & 0,181 & $-0,0013$ \\
\hline Asylberechtigt & $-0,000$ & 0,288 & $-0,0000$ \\
\hline Flüchtling & 0,016 & 0,496 & 0,0028 \\
\hline EU-Ausländer & 0,074 & 0,216 & 0,0134 \\
\hline Nicht-EU-Ausländer & $-0,160$ & 0,154 & $-0,0260$ \\
\hline Hauptschule & $-0,006$ & 0,139 & $-0,0010$ \\
\hline Realschule & 0,070 & 0,138 & 0,0123 \\
\hline Fachhochschulreife / Abitur & 0,115 & 0,192 & 0,0212 \\
\hline Berufsausbildung & $0,309 * *$ & 0,141 & 0,0611 \\
\hline $\begin{array}{l}\text { Prozentualer Anteil in Beschäftigung im 1. Quartal } \\
\text { vor Eintritt in Sozialhilfe }\end{array}$ & $1,080 * * *$ & 0,280 & 0,1874 \\
\hline $\begin{array}{l}\text { Prozentualer Anteil in Beschäftigung im 2. Quartal r } \\
\text { vor Eintritt in Sozialhilfe }\end{array}$ & 0,237 & 0,347 & 0,0411 \\
\hline $\begin{array}{l}\text { Prozentualer Anteil in Beschäftigung im 3. Quartal } \\
\text { vor Eintritt in Sozialhilfe }\end{array}$ & 0,038 & 0,395 & 0,0065 \\
\hline $\begin{array}{l}\text { Prozentualer Anteil in Beschäftigung im 4. Quartal } \\
\text { vor Eintritt in Sozialhilfe }\end{array}$ & $0,483 *$ & 0,278 & 0,0837 \\
\hline Konstante & $-2,049 * * *$ & 0,557 & \\
\hline
\end{tabular}

Loglikelihood: -672,60. Personen, die mehrere IEB-Konten aufweisen, wurden entsprechend gewichtet.

$* \mathrm{p}<0,1, * * \mathrm{p}<0,05, * * * \mathrm{p}<0,01$

Tabelle 3: Matching-Resultate

\begin{tabular}{|l|c|c|c|c|c|}
\hline & Teilnehmer & Nichtteilnehmer & Differenz & Standardfehler & t-Wert \\
\hline Unmatched & 0,108 & 0,124 & $-0,016$ & 0,026 & $-0,62$ \\
\hline Matched & 0,109 & 0,074 & 0,035 & 0,036 & 0,97 \\
\hline
\end{tabular}

Für 188 von 189 Teilnehmern wurde ein passender Nichtteilnehmer gefunden. Differenzen in den beobachtbaren Variablen vor und nach dem Matching sind in Tabelle A1 berichtet. 
Tabelle 4: Bivariates Probit-Modell: Beschäftigungswahrscheinlichkeit 12 Monate nach Eintritt in das Programm

\begin{tabular}{|c|c|c|c|}
\hline Variable & Koeffizient & $\begin{array}{c}\text { Robuster } \\
\text { Standardfehler }\end{array}$ & $\begin{array}{l}\text { Marginaler } \\
\text { Effekt }\end{array}$ \\
\hline Teilnahme am Programm (D=1) & 1,017 & 0,636 & 0,2736 \\
\hline Alter & 0,029 & 0,027 & 0,0056 \\
\hline Geschlecht (Mann=1) & $0,229 * *$ & 0,114 & 0,0446 \\
\hline Verheiratet & $-0,255$ & 0,168 & $-0,0446$ \\
\hline $\begin{array}{l}\text { Stellung im Haushalt: HH Vorstand oder } \\
\text { Ehepartner }\end{array}$ & $-0,125$ & 0,164 & $-0,0232$ \\
\hline Stellung im Haushalt: Kind & 0,042 & 0,188 & 0,0084 \\
\hline Asylberechtigt & 0,055 & 0,286 & 0,0110 \\
\hline Flüchtling & 0,070 & 0,445 & 0,0141 \\
\hline EU-Ausländer & 0,133 & 0,242 & 0,0275 \\
\hline Nicht-EU-Ausländer & $-0,085$ & 0,162 & $-0,0160$ \\
\hline Hauptschule & $-0,033$ & 0,149 & $-0,0063$ \\
\hline Realschule & 0,100 & 0,146 & 0,0199 \\
\hline Fachhochschulreife / Abitur & 0,218 & 0,198 & 0,0465 \\
\hline Berufsausbildung & $0,281^{*}$ & 0,147 & 0,0606 \\
\hline \% in Beschäftigung im 1. Quartal vor Eintritt & $0,959 * * *$ & 0,322 & 0,1855 \\
\hline \% in Beschäftigung im 2. Quartal vor Eintritt & 0,233 & 0,386 & 0,0451 \\
\hline \% in Beschäftigung im 3. Quartal vor Eintritt & 0,148 & 0,415 & 0,0286 \\
\hline \% in Beschäftigung im 4. Quartal vor Eintritt & $0,487 *$ & 0,284 & 0,0942 \\
\hline Konstante & $-2,287 * * *$ & 0,576 & \\
\hline
\end{tabular}




\section{Anhang}

Tabelle A1: Unterschiede in beobachteten Variablen vor und nach dem Matching

\begin{tabular}{|c|c|c|c|c|c|c|c|}
\hline \multirow[b]{2}{*}{ Variable } & \multirow[b]{2}{*}{ Sample } & \multicolumn{2}{|c|}{ Mittelwert } & \multirow[b]{2}{*}{$\%$ Bias } & \multirow{2}{*}{$\begin{array}{c}\text { \%Bias- } \\
\text { Reduktion }\end{array}$} & \multirow[b]{2}{*}{$\mathrm{t}$} & \multirow[b]{2}{*}{$\mathrm{p}>|\mathrm{t}|$} \\
\hline & & Teilnehmer & $\begin{array}{l}\text { Nicht- } \\
\text { teilnehmer }\end{array}$ & & & & \\
\hline Alter & $\begin{array}{r}\text { Unmatchhed } \\
\text { Matched }\end{array}$ & $\begin{array}{l}20,545 \\
20,553\end{array}$ & $\begin{array}{l}21,303 \\
20,697\end{array}$ & $\begin{array}{l}-35,6 \\
-6,7\end{array}$ & 81,0 & $\begin{array}{l}-4,17 \\
-0,71\end{array}$ & $\begin{array}{l}0,000 \\
0,480\end{array}$ \\
\hline Geschlecht & $\begin{array}{r}\text { Unmatchhed } \\
\text { Matched }\end{array}$ & $\begin{array}{l}0,58201 \\
0,57979\end{array}$ & $\begin{array}{l}0,47629 \\
0,56383 \\
\end{array}$ & $\begin{array}{c}21,3 \\
3,2\end{array}$ & 84,9 & $\begin{array}{l}2,66 \\
0,31\end{array}$ & $\begin{array}{l}0,008 \\
0,755\end{array}$ \\
\hline Verheiratet & $\begin{array}{r}\text { Unmatchhed } \\
\text { Matched }\end{array}$ & $\begin{array}{l}0,04233 \\
0,04255\end{array}$ & $\begin{array}{l}0,20151 \\
0,03723\end{array}$ & $\begin{array}{c}-50,1 \\
1,7\end{array}$ & 96,7 & $\begin{array}{c}-5,32 \\
0,26\end{array}$ & $\begin{array}{l}0,000 \\
0,793\end{array}$ \\
\hline $\begin{array}{l}\text { Stellung HH } \\
\text { Vorstand / Ehep. }\end{array}$ & $\begin{array}{r}\text { Unmatchhed } \\
\text { Matched }\end{array}$ & $\begin{array}{l}0,32275 \\
0,31915\end{array}$ & $\begin{array}{l}0,17457 \\
0,31383\end{array}$ & $\begin{array}{c}34,7 \\
1,2\end{array}$ & 96,4 & $\begin{array}{l}4,69 \\
0,11 \\
\end{array}$ & $\begin{array}{l}0,000 \\
0,912 \\
\end{array}$ \\
\hline $\begin{array}{l}\text { Stellung im } \\
\text { HH: Kind }\end{array}$ & $\begin{array}{r}\text { Unmatchhed } \\
\text { Matched }\end{array}$ & $\begin{array}{l}0,04233 \\
0,04255\end{array}$ & $\begin{array}{l}0,11422 \\
0,03723\end{array}$ & $\begin{array}{c}-27,0 \\
2,0\end{array}$ & 92,6 & $\begin{array}{c}-2,99 \\
0,26\end{array}$ & $\begin{array}{l}0,003 \\
0,793\end{array}$ \\
\hline Asylberechtigt & $\begin{array}{r}\text { Unmatchhed } \\
\text { Matched }\end{array}$ & $\begin{array}{l}0,01587 \\
0,01596\end{array}$ & $\begin{array}{l}0,04634 \\
0,00532\end{array}$ & $\begin{array}{c}-17,6 \\
6,1\end{array}$ & 65,1 & $\begin{array}{c}-1,92 \\
1,00\end{array}$ & $\begin{array}{l}0,055 \\
0,316\end{array}$ \\
\hline Flüchtling & $\begin{array}{r}\text { Unmatchhed } \\
\text { Matched }\end{array}$ & $\begin{array}{l}0,01587 \\
0,01596\end{array}$ & $\begin{array}{c}0,01616 \\
0,0266\end{array}$ & $\begin{array}{l}-0,2 \\
-8,5\end{array}$ & $-3558,6$ & $\begin{array}{l}-0,03 \\
-0,71\end{array}$ & $\begin{array}{l}0,977 \\
0,476\end{array}$ \\
\hline EU-Ausländer & $\begin{array}{r}\text { Unmatchhed } \\
\text { Matched }\end{array}$ & $\begin{array}{l}0,08995 \\
0,08511\end{array}$ & $\begin{array}{l}0,06789 \\
0,07979\end{array}$ & $\begin{array}{l}8,2 \\
2,0\end{array}$ & 75,9 & $\begin{array}{l}1,07 \\
0,19\end{array}$ & $\begin{array}{l}0,284 \\
0,852\end{array}$ \\
\hline $\begin{array}{l}\text { Nicht-EU- } \\
\text { Ausländer }\end{array}$ & $\begin{array}{r}\text { Unmatchhed } \\
\text { Matched }\end{array}$ & $\begin{array}{l}0,14286 \\
0,14362\end{array}$ & $\begin{array}{l}0,20474 \\
0,14894\end{array}$ & $\begin{array}{l}-16,4 \\
-1,4\end{array}$ & 91,4 & $\begin{array}{l}-1,96 \\
-0,15\end{array}$ & $\begin{array}{l}0,050 \\
0,884\end{array}$ \\
\hline Hauptschule & $\begin{array}{r}\text { Unmatchhed } \\
\text { Matched } \\
\end{array}$ & $\begin{array}{l}0,38624 \\
0,38298 \\
\end{array}$ & $\begin{array}{l}0,24030 \\
0,40426 \\
\end{array}$ & $\begin{array}{l}31,8 \\
-4,6 \\
\end{array}$ & 85,4 & $\begin{array}{r}4,17 \\
-0,42 \\
\end{array}$ & $\begin{array}{l}0,000 \\
0,674 \\
\end{array}$ \\
\hline Realschule & $\begin{array}{r}\text { Unmatchhed } \\
\text { Matched }\end{array}$ & $\begin{array}{l}0,20635 \\
0,20745\end{array}$ & $\begin{array}{l}0,30927 \\
0,18617\end{array}$ & $\begin{array}{c}-23,7 \\
4,9\end{array}$ & 79,3 & $\begin{array}{c}-2,84 \\
0,52\end{array}$ & $\begin{array}{l}0,005 \\
0,605\end{array}$ \\
\hline $\begin{array}{l}\text { Fachhochschulreife } \\
\text { / Abitur }\end{array}$ & $\begin{array}{r}\text { Unmatchhed } \\
\text { Matched }\end{array}$ & $\begin{array}{l}0,04762 \\
0,04787\end{array}$ & $\begin{array}{l}0,11746 \\
0,04787\end{array}$ & $\begin{array}{c}-25,6 \\
0,0\end{array}$ & 100 & $\begin{array}{c}-2,85 \\
0,00\end{array}$ & $\begin{array}{l}0,004 \\
1,000\end{array}$ \\
\hline Berufsausbildung & $\begin{array}{r}\text { Unmatchhed } \\
\text { Matched }\end{array}$ & $\begin{array}{l}0,06349 \\
0,06383\end{array}$ & $\begin{array}{l}0,17888 \\
0,06915\end{array}$ & $\begin{array}{l}-35,9 \\
-1,7\end{array}$ & 95,4 & $\begin{array}{l}-3,97 \\
-0,21\end{array}$ & $\begin{array}{l}0,000 \\
0,837\end{array}$ \\
\hline $\begin{array}{l}\text { Beschäftigung } 1 . \\
\text { Quartal vor Eintritt }\end{array}$ & $\begin{array}{r}\text { Unmatchhed } \\
\text { Matched }\end{array}$ & $\begin{array}{l}0,01712 \\
0,01721\end{array}$ & $\begin{array}{l}0,07006 \\
0,02262\end{array}$ & $\begin{array}{l}-31,4 \\
-3,2\end{array}$ & 89,8 & $\begin{array}{l}-3,18 \\
-0,49\end{array}$ & $\begin{array}{l}0,002 \\
0,628\end{array}$ \\
\hline $\begin{array}{l}\text { Beschäftigung } 2 . \\
\text { Quartal vor Eintritt }\end{array}$ & $\begin{array}{r}\text { Unmatchhed } \\
\text { Matched }\end{array}$ & $\begin{array}{l}0,03122 \\
0,03139\end{array}$ & $\begin{array}{l}0,11498 \\
0,04945 \\
\end{array}$ & $\begin{array}{l}-35,6 \\
-7,7\end{array}$ & 78,4 & $\begin{array}{l}-3,81 \\
-1,01\end{array}$ & $\begin{array}{l}0,000 \\
0,311\end{array}$ \\
\hline $\begin{array}{l}\text { Beschäftigung } 3 . \\
\text { Quartal vor Eintritt }\end{array}$ & $\begin{array}{r}\text { Unmatchhed } \\
\text { Matched } \\
\end{array}$ & $\begin{array}{l}0,04378 \\
0,04401 \\
\end{array}$ & $\begin{array}{l}0,12674 \\
0,05676 \\
\end{array}$ & $\begin{array}{c}-32,4 \\
-5,0 \\
\end{array}$ & 84,6 & $\begin{array}{l}-3,55 \\
-0,62 \\
\end{array}$ & $\begin{array}{l}0,000 \\
0,537 \\
\end{array}$ \\
\hline $\begin{array}{l}\text { Beschäftigung } 4 . \\
\text { Quartal vor Eintritt }\end{array}$ & $\begin{array}{r}\text { Unmatchhed } \\
\text { Matched }\end{array}$ & $\begin{array}{l}0,06617 \\
0,06513\end{array}$ & $\begin{array}{l}0,13691 \\
0,06750\end{array}$ & $\begin{array}{l}-25,1 \\
-0,8\end{array}$ & 96,6 & $\begin{array}{l}-2,84 \\
-0,10\end{array}$ & $\begin{array}{l}0,005 \\
0,921\end{array}$ \\
\hline
\end{tabular}

Leichte Abweichungen in den Werten für Personen vor dem Matching im Vergleich zu den in Tabelle 1 berichteten Werten treten aufgrund von fallweisem Ausschluss bei fehlenden Werten einzelner Variablen im Matchingverfahren auf. 
Tabelle A2: Bivariates Probit-Modell: Eintrittswahrscheinlichkeit in das Programm

\begin{tabular}{|l|c|c|}
\hline \multicolumn{1}{|c|}{ Variable } & Koeffizient & $\begin{array}{c}\text { Robuster } \\
\text { Standardfehler }\end{array}$ \\
\hline Programmbelegung & $-0,029^{* * *}$ & 0,005 \\
Alter & 0,015 & 0,024 \\
Geschlecht (Mann=1) & 0,008 & 0,106 \\
Verheiratet & $-0,903^{* * *}$ & 0,208 \\
Stellung im Haushalt: HH Vorstand oder & $0,389^{* * *}$ & 0,133 \\
Ehepartner & $-0,526^{* *}$ & 0,214 \\
Stellung im Haushalt: Kind & $-0,614^{* *}$ & 0,311 \\
Asylberechtigt & 0,1484 & 0,396 \\
Flüchtling & $0,474^{* *}$ & 0,208 \\
EU-Ausländer & $-0,113$ & 0,159 \\
Nicht-EU-Ausländer & $0,433^{* * *}$ & 0,133 \\
Hauptschule & $-0,113$ & 0,140 \\
Realschule & $-0,172$ & 0,218 \\
Fachhochschulreife / Abitur & $-0,517 * *$ & 0,188 \\
Berufsausbildung & $-1,190^{* * *}$ & 0,417 \\
\% in Beschäftigung im 1. Quartal vor Eintritt & $-0,525$ & 0,627 \\
\% in Beschäftigung im 2. Quartal vor Eintritt & $-0,259$ & 0,569 \\
\% in Beschäftigung im 3. Quartal vor Eintritt & 0,020 & 0,308 \\
\% in Beschäftigung im 4. Quartal vor Eintritt & $1,567^{* *}$ & 0,700 \\
Konstante & $-0,541$ & 0,31 \\
Rho & & \\
\hline
\end{tabular}

$* \mathrm{p}<0,1, * * \mathrm{p}<0,05, * * * \mathrm{p}<0,01$ 